\title{
Infrastrukturen als Produkte und Produzenten von Zeit
}

\author{
Jens Ivo Engels
}

Technical Infrastructures as Products and Producers of Time

During the last few years "time" has been an intensively debated topic among historians. This concept is increasingly being considered in the history of technology as well. For historical research on infrastructures, however, time still plays a minor role. This article takes up recent approaches and presents the relationship between networked infrastructures and time as reciprocal: In infrastructures, different epochs with their temporal contexts are deposited as layers of time. This is reflected not only in technical components of different ages, but also in organizational structures or action routines. Infrastructures produce time by influencing the ways persons, information or goods circulate through them. In this paper the use of the following metaphors for the analysis of these relationships are proposed: "time layer" and "palimpsest" for the consequences of their historicity materialized in the systems, "inscription" and "overwriting" for the underlying processes. The production of temporality by the infrastructures thus composed will be described as the "rhythms" that structure circulation. In sum, a complex picture emerges: the different time layers of an infrastructure contribute to the formation of rhythms.

Keywords: Infrastructure, Time, Time layers, Production of time, Rhythms

"Zeit” ist seit einigen Jahren ein intensiv debattiertes Thema in der Geschichtswissenschaft. Auch in der Technikgeschichte finden zunehmend Überlegungen dazu statt. In den historischen Forschungen zu Infrastrukturen spielt der Aspekt allerdings noch eine geringe Rolle. In diesem Aufsatz möchte ich die jüngsten Ansätze aufgreifen und das Verhältnis netzgebundener Infrastrukturen zur Zeit als ein doppelseitiges Produktionsverhältnis darstellen: In Infrastrukturen lagern sich unterschiedliche Epochen mit ihren zeitlichen Kontexten als Zeitschichten ab. Dies schlägt sich nicht nur in technischen Komponenten unterschiedlichen Alters nieder, sondern auch in Organisationsstrukturen oder Handlungsroutinen. Zugleich produzieren Infrastrukturen aber auch Zeit, indem sie Art und Geschwindigkeit beeinflussen, mit der Stoffe, Personen, Informationen oder Güter durch sie zirkulieren. In diesem Aufsatz schlage ich die Nutzung von folgenden Metaphern für die Analyse dieser Zusammenhänge vor: „Zeitschicht" und „Palimpsest” für die in den Systemen materialisierten Folgen ihrer Geschichtlichkeit, „Einschreibung" und „Überschreibung" für die zugrundeliegenden Prozesse. Die Produktion von Zeitlichkeit durch die so zusammengesetzten Infrastrukturen soll in Form von „Rhythmen” beschrieben werden, welche die Zirkulation strukturieren. In der Summe ergibt sich ein komplexes Bild: Die unterschiedlichen Zeitschichten einer Infrastruktur tragen zur Ausbildung von Rhythmen bei.

Schlüsse/wörter: Infrastruktur, Zeit, Zeitschichten, Zeitproduktion, Rhythmen

In diesem Artikel wird die Dimension der Zeitlichkeit technischer Infrastrukturen exploriert. Dabei geht es nicht um neue empirische Ergebnisse, sondern um konzeptionelle Überlegungen. Zunächst steht die Infrastruktur als Zeitspeicher im Mittelpunkt. Nach einer Einführung zur Zeitforschung in der Geschichtswissenschaft wird das Konzept der Pfadabhän- 
gigkeit kritisch gewürdigt, dem oft eine zu einfache Vorstellung von Temporalstrukturen zugrunde liegt. Anschließend werden vier Begriffe bzw. Konzepte vorgestellt, die die historische Analyse von Zeitspeicherung in Infrastrukturen ermöglichen. Zunächst wird auf das Resultat fokussiert: Über lange Zeiträume gewachsene Systeme weisen Zeitschichten auf und können als Palimpseste analysiert werden. Anschließend rücken Vorgänge in den Fokus, die zu diesen Resultaten führten, namentlich „Einschreibung“ und „Überschreibung“. Im nächsten Schritt wird der Blick umgekehrt: Zur Zeitlichkeit von Infrastrukturen gehört nicht nur Zeitspeicherung. Denn daneben produzieren Infrastrukturen auch Zeit oder beeinflussen die Zeitwahrnehmung und -nutzung. Um diesen Effekt zu beschreiben wird das Konzept der Rhythmusanalyse vorgestellt. Am Schluss wird anhand von Beispielen erläutert, wie man beide Untersuchungsrichtungen zusammenführen kann: Unterschiedliche Zeitschichten innerhalb eines technischen Systems beeinflussen seine Rhythmen. Die Produktion von Zeit durch Infrastrukturen ist also abhängig von ihren Zeitschichten. Infrastrukturen sind Produkte und Produzenten von Zeit.

Wenn im Folgenden von Infrastrukturen die Rede ist, dann sind technische, netzgebundene Systeme gemeint, die der Zirkulation von Personen, Gütern, Energie oder Informationen dienen. Diese bestehen nicht nur aus technologischen Komponenten. Dazu gehören auch die rechtlichen, ökonomischen, kulturellen, sozialen, organisatorischen Grundlagen und Rahmenbedingungen: In den Infrastrukturen manifestieren sich Austauschprozesse zwischen sozialer und materieller Welt (Laak 2001; Dolata \& Werle 2007; Bijker et al. 1987).

Vorweggeschickt sei, dass in diesem Aufsatz mit Metaphern operiert wird. Metaphern erzeugen im Idealfall ein starkes Bild, indem sie komplexe Zusammenhänge auf einen Begriff bringen. Der Nachteil von Metaphern liegt darin, dass sie wegen ihrer Assoziationswirkung häufig unpräzise sind. Allerdings operieren sehr viele Arbeiten zur Zeit mit Metaphern und plastischen Begriffen. Da Zeit und Temporalität auf hohem Abstraktionsniveau verhandelt werden, müssen diese Nachteile in Kauf genommen werden. Man läuft sonst Gefahr, keinen handhabbaren sprachlichen Ausdruck für die zu beschreibenden Phänomene zu finden. Abstrakte Benennungen wie „Polychronie“ oder „Pluritemporalität“ (Rothauge 2017: 735; Landwehr 2017: 240) erschließen sich dagegen nur mühsam. Daher wird hier auch darauf verzichtet, zwischen Zeit und Temporalität zu trennen, oder gar mit festen Definitionen beider Begriffe zu arbeiten.

$\mathrm{Zu}$ den Merkmalen von Metaphern gehört es auch, dass man sich womöglich nicht auf einen einzelnen Begriff festlegen muss, sondern mit mehreren gleichzeitig arbeiten kann. Dies ist immer dann sinnvoll, wenn mehrere Metaphern es erlauben, unterschiedliche Merkmale des Bezeich- 
neten zum Ausdruck zu bringen, was ein einzelner Begriff nicht leistet. Gut geeignet sind die Begriffe „Zeitschichten“ sowie „Palimpsest". Ich möchte das im Folgenden kurz ausführen.

Hierzu zählt zunächst eine grundlegende Differenzierung: „Zeitscheibe“ benennt einen synchronen Zusammenhang, gleichsam die Einheit einer Epoche, wenn man so will, eine vertikale Zeit. Dagegen verweist das Bild der „Zeitschichten“ auf horizontal übereinander gelagerte Zeiten, auf eine Vielzahl von Zeitscheiben, welche sich übereinandergelegt haben.

\section{Zeit und Zeitlichkeit als neues Forschungsfeld}

Wenn in historischer Perspektive über Infrastrukturen und Zeit geforscht wurde, dann beschränkte sich dies bislang meist auf die Durchsetzung der Uhrenzeit und auf die allgemeine Beschleunigung des Lebens im technologischen Zeitalter (Laak 2018; Richter 2018). Die Erfindung der modernen Uhrenzeit als globales System mit weltweit definierten Zeitzonen gilt als Ergebnis der Transportinfrastruktur, vor allem der Eisenbahn. Man spricht auch von „Eisenbahnzeit“. Um Fahrpläne aufeinander abstimmen zu können, bot sich ein universelles Zeitmessungssystem an. Aus dieser Perspektive war der Siegeszug der Eisenbahn der Ursprung der Weltzeitmessung (Blaise 2000). In der Forschung ging man lange davon aus, dass sich diese von Ingenieuren ersonnene Standardzeit schnell auf den Alltag aller Menschen in westlichen Gesellschaften auswirkte. Damit einher ging die allgemeine Beschleunigung (etwa Zoellner 2014: 275; Schivelbusch 1977; Thompson 1967). Jüngste Forschungen relativieren diesen Eindruck: Die Aneignungsprozesse waren viel komplexer. Neben hegemonialen Zeitkonzepten gab es partielle Verweigerung und individuell sehr unterschiedliche Erfahrungen (Zimmer 2019; Ogle 2015; Sprute 2015).

Während aber die Erfindung einer einheitlichen Zeit, Beschleunigung und Aneignungsprozesse untersucht wurden, gibt es noch kaum Studien darüber, wie sich Zeit in Infrastrukturen manifestiert. Das hat unterschiedliche Gründe. Der erste Grund liegt darin, dass das Hauptinteresse der historischen Infrastrukturforschung auf der Raumwirkung lag, nicht auf Temporalität (etwa Laak 2004; Höhne 2017). Vor allem die geographischpolitische Integrationswirkung von Netzen stand lange Zeit im Mittelpunkt der Aufmerksamkeit (Schiefelbusch \& Dienel 2014; Högselius et al. 2015; Ambrosius \& Henrich-Franke 2013; Mattelart 2000).

Hinzu kommt ein weiterer Grund. Über viele Jahrzehnte war Zeitlichkeit im avancierten methodischen Sinn kein Thema von Historikerinnen und Historikern. Zwar galt die Aufmerksamkeit der Historiker schon immer 
der Epocheneinteilung. Komplexere Fragen etwa nach der „Gleichzeitigkeit des Ungleichzeitigen“"wurden zwar von einzelnen Vertretern wie Reinhart Koselleck adressiert, aber eine breitere Debatte dazu hat es lange nicht gegeben. Die Diskussion war beherrscht von eher normativen Fragen nach Vorreitern und Nachzüglern im Prozess der Modernisierung (vgl. Landwehr 2012). In den meisten historischen Arbeiten erschien Zeit synonym mit der (vorgeblich objektiven) Uhrenzeit.

Das änderte sich erst in den letzten Jahren (Esposito 2017; Geppert \& Kössler 2015; Patzel-Mattern \& Franz 2015). Das neue Interesse an der Zeit gab rasch Anlass für die Ausrufung eines temporal turn in der Geschichtswissenschaft (Rothauge 2017). Jetzt wurde anerkannt: Unterschiedliche Zeitkonzepte und Zeitempfindungen prägten die historische Wirklichkeit - und unterschiedliche Gesellschaften und Epochen schufen unterschiedliche und oft miteinander konkurrierende Zeitregime (Fryxell 2019). Im Zuge dieser Perspektivänderung setzte sich durch, was auch zu den Prämissen des vorliegenden Aufsatzes gehört: Zeit sollte stets im Plural gedacht werden.

Die Geschichte der Temporalitäten, so Matthew S. Champion erst jüngst in einem Themenheft zu diesem Thema, behandele die „relationship between measurement, social form and cultural norms, expectations, and experiences of time“ (Champion 2019: 249). Sie frage danach, wie zeitliche Strukturen und Normen Kommunikation, soziale Praktiken und Kulturen beeinflussen (ebd. 253).

In der Sozial- und Kulturwissenschaft gibt es schon etwas länger dieses Interesse an komplexen Zeitkonzepten. Konsens ist seit den 1990er Jahren, dass Zeit und Raum gemeinsam sozial konstruiert sind (Adam 1990; Nowotny 1992; May \& Thrift 2001). Bis auf die 1920er Jahre gehen Überlegungen über die Unterschiede zwischen objektivierbaren und subjektiven Konzepten von Zeitmessung zurück, teilweise beschrieben als ein Gegensatz von "linearer“ und "relationaler“ oder „zyklischer“ Zeit (McTaggart 1927; Landwehr 2017).

Erste Versuche mit diesem neuen Blick auf die Technikgeschichte zu schauen verdanken wir Heike Weber. Sie betont vor allem das Fortleben des Bestehenden und alter Technologie in späteren Phasen der Technikentwicklung. Generell plädiert sie für eine Abkehr vom Paradigma der Innovationsgeschichte, hin zu Prozessen der Transition. Hinzu kommt die Betonung von Phänomenen der „Exnovation“ (Weber 2019: 138). Damit öffnet sie ein neues Feld der Technikgeschichte, nämlich unterschiedlichen zeitlichen Strukturen oder auch Zeitschichten Aufmerksamkeit zu widmen. In diese Forschungsrichtung passt auch das Interesse für die reemersion älterer Technologien (Moss 2017: 159; vgl. Simmie 2012). 
Im Bereich der nicht-historischen Forschung zu Infrastrukturen sind ähnliche Ansätze schon etwas länger in der Diskussion. Infrastrukturen sind in hohem Maß durch Funktionsstörung, Ausfall und Reparatur geprägt. Daraus folgt, dass Dynamik und Veränderungen, nicht aber Statik ihre Wirklichkeit prägen (Graham \& Thrift 2007; Perrow 1984). Alterungsprozesse werden mittlerweile als selbstverständliche Merkmale von Infrastrukturen beschrieben (Doyle \& Betti 2010; Little 2002).

Technik wird in der aktuellen Forschung also nicht nur weniger teleologisch gedacht. Vor allem werden komplizierte Temporalstrukturen adressiert, die in Technik eingeschrieben sind. In diese Richtung argumentiert auch der vorliegende Beitrag.

\section{Pfadabhängigkeit revisited}

Infrastrukturen sind Ergebnisse und Orte der Interaktion von Menschen. Im Fall der großtechnischen Systeme in Industriegesellschaften sind Infrastrukturen Symbole und Manifestationen eines Lebensstils, Materialisierung von Gesellschaftsentwürfen, sind Rückgrat der Ökonomie und Feld von Konflikten. Kurz: Infrastrukturen bilden die Signatur der Industriemoderne - und dies bis in die heutige Zeit. Dirk van Laak hat dies eindrücklich in seinen vielen Arbeiten gezeigt (zuletzt: Laak 2018). Wenn sich eine ganze Epoche im Modus der technischen Infrastruktur ausdrückt (Richter 2018), dann prägt sich umgekehrt auch die Epoche in der Infrastruktur ein.

Insofern sind technische Infrastrukturen Speicher historischer Verhältnisse. Infrastrukturen sind Machtspeicher, denn Gestalt und Ort einer Infrastruktur sind das Ergebnis des Gestaltungswillens der jeweils Herrschenden (Engels \& Schenk 2014). Die Persistenz der Infrastrukturen über Regierungswechsel und Revolutionen hinweg perpetuiert diese Gestaltung, sei es das segregierende System der Wagenklassen in Fernzügen, seien es die Trassenverläufe von Verkehrs- und Versorgungsinfrastrukturen, die ganze Regionen dauerhaft an- oder ausschließen (Laak 2018: 137-149). Auch wenn sie selbst nicht mehr in Nutzung sind, prägen Infrastrukturen städtische Räume über eine lange Zeit (Matern 2016).

Ähnlich argumentieren auch die klassischen Arbeiten zu großtechnischen Systemen: Nach einer Aufbauphase mit hohem Innovationsgrad sind diese technisch, politisch und ökonomisch stabilisiert (Hughes 1983; Melosi 2000). Später werden Veränderungen unwahrscheinlicher, so dass sich die Dominanz einer technischen Lösung gegenüber anderen dauerhaft verfestigt. Auch auf diese Weise speichert ein großtechnisches System ökonomische Verhältnisse und den technischen Wissensstand einer Epoche. 
Das in der bisherigen Forschung wohl wichtigste Paradigma zur Beschreibung dieser langen Zeitdimension ist das der Pfadabhängigkeit. Es beschreibt, wie in der Vergangenheit getroffene Entscheidungen spätere Entwicklungen vorprägen. Das Konzept hilft zu erklären, warum beispielsweise die Achse der Rue de la Cité und der Rue St. Jacques in Paris genau auf der Trasse des Cardo im antiken Lutetia verläuft (vgl. Laak 2018: 207-208 mit weiteren Beispielen). Während sich Trassenverläufe in vielen Fällen (auch) naturräumlich erklären lassen, berücksichtigt das Konzept der Pfadabhängigkeit gesellschaftliche Zusammenhänge. Insbesondere in der Frühzeit dieser Debatte ging es darum zu verstehen, warum selbst unpraktische oder ineffiziente Lösungen nicht ersetzt wurden (David 1986). Häufig wird Pfadabhängigkeit als Einschränkung von Entwicklungsmöglichkeiten beschrieben. Als Erklärung dienten die ökonomischen oder auch gesellschaftlichen Kosten: Für eine Pfadänderung seien sie zu hoch (vgl. Mahoney 2000; Beyer 2006), so dass alternative Entwicklungen nicht stattfanden. Technische und organisatorische Standardisierungen sind ein $\mathrm{Pa}-$ radebeispiel für diesen Zusammenhang - je mehr Akteure daran beteiligt oder technische Systeme davon betroffen sind, umso unwahrscheinlicher eine Pfadabweichung, so zeigen es etwa Beispiele aus dem Eisenbahn- und Telekommunikationswesen seit dem 19. Jahrhundert (Ambrosius \& Henrich-Franke 2015).

Dabei greift das ursprüngliche Modell der Pfadabhängigkeit zu kurz. Denn häufig wird davon ausgegangen, dass ein bestimmter Moment in der Geschichte, eine einzelne Zeitscheibe, während der gesamten Lebensdauer eines Infrastruktursystems dominiert. So verstandene Pfadabhängigkeit suggeriert, dass zu Beginn der Entwicklung eines großtechnischen Systems in gewisser Weise „die Uhren angehalten“ werden. Häufig ist von einem lock-in die Rede, in dem eine Technologie auf Dauer geprägt werde. Das alte Modell der Pfadabhängigkeit war geprägt von einer einfachen Temporalstruktur: Bestehendes schien in einer Art Zeitkapsel konserviert, während die Welt sich linear weiterentwickelte. Das ist zwar in vielen Fällen empirisch gut begründet. Doch diese Vorstellung erlaubt es nicht ausreichend, die Vielzahl der Einflüsse zu berücksichtigen, die in den folgenden Zeitabschnitten auf Infrastruktursysteme einwirken (Maassen 2012).

Die Debatte ist mittlerweile weiter (Schott 2018). Die Entwicklung der Technik ist in Wirklichkeit selten linear, sondern beinhaltet Umwege, Nebenwege und Abweichungen, Verluste und das Wiederauftauchen von Technologien (Weber 2019; Moss 2016b). Timothy Moss hat dieses Problem aufgegriffen und schlägt vor, netzgebundene Infrastrukturen mit dem Konzept der „Assemblage“ zu beschreiben (Moss 2016a: 251-254). Dieser Begriff ist in der Stadtforschung schon länger bekannt (McFarlane 2011; Farías \& Bender 2010). Demnach ist jedes technische System das Resultat 
dynamischer Interaktion von Technologie und gesellschaftlichen Praktiken, und zwar kontinuierlich und im längeren Zeitverlauf. So werden die jeweils herrschenden politischen, sozialen und technologischen Zustände laufend in das System eingeschrieben, wenn auch mit unterschiedlicher Bedeutung. Grundentscheidungen vom Beginn der Entwicklung, etwa die Anlage des radialen Eisenbahnnetzes in Frankreich im 19. Jahrhundert, haben in vielen Fällen mehr Gewicht als spätere Einflüsse. Letztere können aber auch durchaus wichtig sein, etwa in Gestalt des transnationalen Anschlusses an die Netze der Nachbarstaaten. Doch nicht immer nimmt die Relevanz im Lauf der Zeit ab. So können für die touristische Umnutzung eines Kanalsystems gerade die älteren Schleusen besonderen Reiz besitzen, weil die Entschleunigung der Freizeitschipper hier noch besser gelingt als im Angesicht hydraulischer Schleusen. Nach derzeitigem Forschungsstand spricht also sehr viel dafür, von der Präsenz unterschiedlicher Zeitschichten in technischen Systemen auszugehen.

\section{Infrastrukturen als heterogene Zeitspeicher: Schichten und Palimpseste}

Viele technische Infrastrukturen entstehen, wachsen und wandeln sich über lange Zeiträume. Daher enthalten sie Elemente aus ganz unterschiedlichen Epochen. Diese Elemente sind das Ergebnis konkreter historischer Kontexte unterschiedlicher Zeitscheiben im historischen Verlauf. Hier soll es darum gehen, „Fortleben“ und anhaltende Relevanz multipler Vergangenheiten in den technischen Systemen zu beschreiben und ernstzunehmen.

Das Konzept der Zeitschichten wurde in die deutschsprachige Geschichtswissenschaft von Reinhart Koselleck (1923-2006) eingeführt, in dem er sich auf die Geologie bezieht. Geologen entwickelten ab dem Ende der Frühen Neuzeit ein Bewusstsein dafür, dass sich der Aufbau der Erde historisch veränderte. Zeitschichten sind also als eine Analogie zu den geologischen Schichten, die sich in erdgeschichtlichen Zeiträumen nach und nach abgelagert haben. Übertragen auf die Geschichtswissenschaft erlaubt dies nach Koselleck „verschiedene zeitliche Ebenen analytisch zu trennen, auf denen sich Personen bewegen, Ereignisse abwickeln“ (Koselleck 2000: 19). Koselleck geht es vor allem um die Zwischenbezüge zwischen linearen und zyklischen Zeitvorstellungen - was uns jedoch von den Infrastrukturen wegführt. Achim Landwehr hat Kosellecks Ansatz kritisch gewürdigt. Dabei formuliert er einen Vorteil des Zeitschichtenmodells klarer als Koselleck selbst: „,Zeitschichten' verweisen, wie ihr geologisches Vorbild, auf 
mehrere Zeitebenen verschiedener Dauer und unterschiedlicher Herkunft, die dennoch gleichzeitig vorhanden und wirksam sind" (Landwehr 2012: 18).

Dieser Zusammenhang gilt insbesondere für Infrastrukturen, die über lange Zeiträume entstehen. In jeder gewachsenen Infrastruktur gibt es einen Funktionszusammenhang, der die Komponenten unterschiedlichen Alters zu einem funktionierenden Gesamtsystem zusammenbindet. Dies gilt vor allem für langlebige Systeme und solche, die über einen längeren Zeitraum auf- und ausgebaut werden. So greifen etwa in der Londoner Untergrundbahn Weichen, Signale, Schienenabschnitte, Zugangswege aus unterschiedlichen Baujahren in einander. Das gilt für die heutige Gegenwart, aber auch für die jeweilige Gegenwart von 1980, 1950, 1930 und so fort. Zu jedem dieser Zeitpunkte waren Komponenten höchst unterschiedlichen Alters miteinander verbunden, die es gemeinsam ermöglichten, den Londoner Stadtraum bequem zu durchfahren (vgl. Gießmann 2013). Dabei ist das Alter eines Stücks Metall weniger bedeutend als die Tatsache, dass diese Komponenten jeweils den Stand der Technik in ihrem Herstellungsjahr abbilden. Das Ergebnis ist ein Sammelsurium unterschiedlicher technologischer Lösungen, die gleichwohl gemeinsam funktionieren - jedenfalls im Idealfall. In der hier gewählten Terminologie würde man den Stand der Technik einer Komponente aus einer Epoche mit dem Bild der Zeitscheibe beschreiben, deren Überlagerung und Zusammenwirken in einem wachsenden System mit dem Bild der Zeitschichten.

Für die Technikgeschichte hat Heike Weber in einem grundlegenden Aufsatz den Begriff der Zeitschichten aufgenommen und fruchtbar gemacht. „Zeitschichten des Technischen“ sind ihr zufolge Zeitdimensionen, „die durch vergangene Techniken, Technikverwendungen oder Technikdiskurse definiert oder geprägt wurden" (Weber 2019: 108). Mit Nachdruck verweist sie darauf, dass stets Techniken unterschiedlichen Alters nebeneinander genutzt werden. In diesem Zusammenhang spricht sie auch von "Polychronie der Technik“ (Weber 2019: 111). Ihr Augenmerk liegt dabei auf der „Persistenz" alter Technik, selbst in Kontexten beschleunigter Innovation (Weber 2019: 119 und öfter). Auch Dirk van Laak benutzt den Begriff „Zeitschicht“ für das Fortleben des Älteren in Infrastrukturen (Laak 2018: 203). Die Besonderheit im Fall der Infrastrukturen liegt vor allem in der fast immer vorhandenen funktionalen Integration der Zeitschichten untereinander.

Das Bild von den Zeitschichten hat den Vorteil, das langsame Aufwachsen eines technischen Systems über verschiedene Epochen hinweg gut zum Ausdruck zu bringen - ganz so wie sich die Sedimente in einem urzeitlichen Meer über Jahrmillionen absetzten und Sandsteinschichten bildeten. Es repräsentiert also die materialisierten Ergebnisse eines Prozesses. Es re- 
flektiert außerdem die Materialität von Infrastrukturen: Die unterschiedlichen Epochen sind ja in den materiellen Komponenten unterschiedlichen Alters präsent - ganz ähnlich wie im Sedimentgestein. Ein letzter Vorteil: Geologische Schichten sind oft verborgen und können nur durch Ausgrabungen ermittelt werden. Auch die Zeitschichten einer Infrastruktur erschließen sich nicht auf den ersten Blick.

Allerdings hat das Bild auch Nachteile: Die Zeitschichten einer Infrastruktur liegen nicht fein säuberlich chronologisch geordnet übereinander, sondern sind ineinander verschlungen. Jüngst erneuerte Schienenstränge können über eine Brücke aus den 1920er Jahren führen. Dieser Nachteil kann durch Hinzunahme einer zweiten Metapher teilweise vermieden werden: das Palimpsest. Ein Palimpsest bezeichnet eine alte Handschrift, die ausradiert wurde, um den wertvollen Träger (Pergament, Papyrus) ein zweites Mal zu benutzen. Da das Radieren meist nicht vollständig gelingt, erhält man ein Dokument mit zwei übereinandergeschriebenen Texten, gelegentlich auch mit drei oder vier. Das Konzept des Palimpsests findet in der Literaturwissenschaft, aber auch in der Stadtforschung und in den Kulturwissenschaften reichlich Verwendung. Beschrieben werden beispielsweise Präsenz und Nachwirkungen der materiellen Hinterlassenschaften von unterschiedlichen politischen Regimen in einer Stadt. So können gesamte Städte als Palimpseste gelesen werden (Binder 2015; Ackermann 2010).

Das lässt sich auch auf die Infrastruktur übertragen. Viele langlebige Infrastrukturen erleben im Lauf ihrer Entwicklung tiefgreifende Umnutzungen: Ein bislang für den Gütertransport genutzter Schifffahrtskanal wandelt sich in eine Infrastruktur des Wassersports. Wie beim Palimpsest schimmert die alte Nutzung hindurch, etwa wenn in einem früheren Warenlager nun die Versorgungsinfrastruktur einer Marina für Freizeitkapitäne untergebracht ist. Auch eine mögliche Parallelnutzung (Tourismus ergänzt den Güterverkehr) kann mit dem Konzept des Palimpsests gut beschrieben werden (zur Kanalgeschichte Roblin 2003; Morin 1994).

Für die digitale Überformung der materiellen Welt in den letzten Jahren hat Mark Graham den Begriff nutzbar gemacht. Am Beispiel der augmented reality in Städten (etwa bei Navigationssystemen) verweist er auf die unterschiedlichen Zeitdimensionen. Während die Nutzer durch die Stadt gehen oder fahren, erhalten sie über ein Navigationsgerät Informationen. Diese Informationen sind notwendigerweise alt, nämlich vor einer bestimmten Zeit ins System eingepflegt worden. Der Zeitpunkt der Eintragung kann Sekunden zurückliegen, aber auch Jahre, je nachdem, wie intensiv das System betreut wird und abhängig davon, in welchem Rhythmus bestimmte Informationen aktualisiert werden. Graham spricht von „Datenschatten“ aus der Vergangenheit. Jeder kennt das Problem, wenn 
er laut Navi in eine Straße einbiegen soll, die vor ein paar Tagen zur Fußgängerzone umgestaltet wurde (Graham 2013). Selbst in der digitalen Epoche, in der die Gegenwart angeblich schrumpft (Lübbe 2000), beeinflussen vergangene Zeitscheiben also die Realität von Technik. Insbesondere die technischen Infrastrukturen sind davon geprägt.

Auch die Metapher vom Palimpsest besitzt Nachteile. Sie geht von einem bewussten Akt des Auslöschens alter Schichten aus - das ist in Infrastrukturen meist nicht der Fall, da man konstruktiv auf Bestehendem aufbaut. Beide Metaphern, Schichten und Palimpseste, haben also Vor- und Nachteile, lassen sich aber gut verbinden - das zeigt der Aufsatz von Graham, der beide verwendet.

\section{Zeitschichten einschreiben und überschreiben}

Beide Metaphern können nicht nur gut miteinander kombiniert werden, sondern sie entsprechen auch zwei Vorgängen: Einschreibung und Überschreibung. Infrastrukturen bestehen auch, aber nicht nur aus materiellen Objekten. Ihre Besonderheit als soziotechnische Systeme liegt in der spezifischen Kombination von materiellen Artefakten (wie etwa Stromleitungen), organisatorischen Einrichtungen (wie Besitzverhältnisse und Verwaltungsstrukturen) sowie menschlichen Praktiken (vom Nutzerverhalten der Berufspendler bis zur korrekten Beachtung von Signalen bei Lokführern) (vgl. Mayntz 1997; Vleuten 2006). Dabei entsteht insgesamt ein Funktionszusammenhang: Güter, Menschen, Energie und Informationen zirkulieren in den Systemen (zur Zirkulation Höhne 2017).

Vergleichsweise einfach ist es, das Baujahr der technischen Komponenten zu ermitteln und ausgehend davon die Zeitschichten einer Infrastruktur zu bestimmen. Darauf kann in bestimmten Fällen mit langfristiger Planung reagiert werden. Ein Beispiel aus dem Verkehrswesen: Der Rijkswaterstaat, die niederländische Verkehrsinfrastrukturbehörde, führt ein Register über das Alter sämtlicher Bauteile in Straßen, Brücken, Tunneln und Kanälen und leitet daraus das Jahr des Renovierungsbedarfs ab. Da die verbauten Materialien unterschiedlich schnell altern, ergibt sich hieraus schon ein dynamisches Bild; manche Bauwerke müssen kurzfristig, andere erst nach längerer Zeit renoviert werden. Daraus folgt derzeit eine Aufgabenplanung bis ins Jahr 2050 (Rijkswaterstaat 2017). ${ }^{1}$ Solche Bedarfsplanungen sind keine niederländische Besonderheit; es gibt sie für viele komplexe Infrastruktursysteme in den Industriestaaten.

Komplizierter wird die Lage, wenn dynamische Einflüsse hinzukommen. Bleiben wir beim Beispiel der Verkehrsinfrastruktur und ihrer Haltbarkeit: 
Die Haltbarkeit eines Bauwerks ist nicht allein von den Materialeigenschaften abhängig, sondern auch von der Nutzung. Wenn die Verkehrsdichte in einem bestimmten Jahrzehnt zu- oder abnimmt, hat dies Konsequenzen für die Dauer der weiteren Nutzung. Man könnte sagen, dass sich in einem solchen Fall unterschiedliche Zeitscheiben in dem Brückenbauwerk einschreiben und als Zeitschichten wirksam werden: die Materialeigenschaften des Betons aus einer Epoche und die Ermüdungsfolgen aus der Nutzungsweise einer anderen Epoche.

Infrastrukturen gelten üblicherweise als Verbindung zwischen Mensch und Umwelt, als Medien zwischen Kultur und Natur (Laak 2001: 370). In der Konsequenz wird nicht nur menschliches Handeln in die Infrastrukturen eingeschrieben, sondern dazu gehören auch Einflüsse aus der Umwelt. Extremereignisse wie Überflutungen oder Erdbeben führen zu Zerstörungen. Oft werden sie mit Reparaturvorgängen beantwortet, die wiederum dauerhaft in den technischen Anlagen selbst, oder aber in Strategien zur Krisenprävention ihren Niederschlag finden (Schenk 2012). Wenn man nach einer Sturmflut systematisch die Deiche erhöht, dann ist das der materielle Ausdruck eines in der Zeit lokalisierten Ereignisses inklusive der gewählten Bewältigungsstrategie. Allerding ist „die Umwelt" mit Ausnahme der Zerstörungswirkung nur vermittelt an der Einschreibung beteiligt: Die Bewältigungsstrategien sind die Folge der materiellen und technischen Möglichkeiten einer Gesellschaft sowie von politisch ausgehandelten Prioritäten. Statt Deicherhöhung wäre auch die Anlage von Poldern denkbar oder die Rückverlegung der Deichlinien ins Inland. Folglich bleibt jeder dieser nichtmenschlichen Faktoren menschlich vermittelt. Unterschiedliche Auffassungen von Naturgefahren und Risiken führen zu unterschiedlichen technischen Lösungen (Bijker 2007).

Nicht allein in materiellen Artefakte und technischen Komponenten finden sich die Sedimente vergangener Epochen. Sie finden sich ebenso in den Grundprinzipien der Organisation von Infrastrukturen, etwa in der Frage nach dezentraler oder zentralisierter Versorgung, privatem oder öffentlichem Besitz an den Einrichtungen (Guldi 2012). Folglich sind auch die Grundsätze im alltäglichen Betriebsablauf Ergebnisse historischer Entscheidungen und damit sedimentierte Zeit: Verkehrsregeln (rechts vor links?) oder die Geltung bestimmter Symbole werden über lange Zeiträume beibehalten und verdauern damit die Wirksamkeit einer unter bestimmten zeitlichen Bedingungen getroffenen Entscheidung.

Die Sedimentierung wirkt auch auf der Ebene von Praktiken: Etwa im Fall des Schleusenwärters, der im frühen 21. Jahrhundert an einer historischen Schleusenanlage beim Aufkurbeln die gleichen Bewegungen ausführt wie sein Vorgänger hundert Jahre vor ihm. Allerdings sind es nicht exakt 
dieselben, wenn er dabei in der anderen Hand sein Smartphone hält und darauf die Zahl der für die nächsten Stunden erwarteten Schiffe abliest.

Das Skript für die Handlungen des Schleusenwärters führt vor Augen, dass es neben dem Einschreiben häufig zu Prozessen des Überschreibens kommt. Wobei wir wieder beim Palimpsest und der Komplexität von Temporalstrukturen wären. Etwa wenn schadhafte Komponenten ausgetauscht werden, ein Verkehrssystem wie die Eisenbahn im Lauf der Geschichte erst privatisiert, dann verstaatlicht, dann wieder reprivatisiert wird. In der Regel werden die vorherigen Zustände nicht komplett ausgelöscht, sondern sie bleiben weiterhin relevant, mehr oder weniger sichtbar. Alte Telefonkabel wurden lange Zeit als Infrastruktur für die neuen Internetverbindungen genutzt (Laak 2018: 208). Auch in der IT-Infrastruktur gilt die Regel, dass ein einmal gewählter Code regelmäßig ergänzt und aktualisiert wird, in den Tiefenstrukturen aber die Ursprungssoftware präsent bleibt, wie etwa in der Software der deutschen Bankhäuser die Programmiersprache COBOL (Weber 2019: 133).

\section{Infrastrukturen als Taktgeber}

Wir haben Infrastrukturen als Produkte komplexer Sedimentation unterschiedlicher Zeitschichten kennengelernt. Die Temporalität der Infrastrukturen beschränkt sich aber nicht darauf. Infrastrukturen sind auch Produzenten von Zeit.

In unserem Alltagsverständnis zeigen Uhren die Zeit an. Wir gehen davon aus, dass Uhren die Zeit nur messen, welche gleichsam objektiv gegeben ist. Die neuere Zeitforschung hat jedoch das Konzept einer „objektiven Zeit" verworfen. In konstruktivistischer Perspektive wird davon ausgegangen, dass Zeit „gemacht" wird, und zwar von ganz unterschiedlichen Taktgebern oder Rhythmen (Adam 1990). Die Bedeutung von Rhythmen für die Entstehung multipler Zeiten ist in der jüngsten Forschung deutlich betont worden (Schmolinsky et al. 2018; vgl. auch Lefebvre 1992). In der Infrastrukturforschung hat das Interesse für Rhythmen gerade erst begonnen (Coletta \& Kitchin 2017).

Es liegt auf der Hand, dass netzgebundene Infrastrukturen stets Rhythmen produzieren. Dies ist schon deshalb so, weil Infrastrukturen Zirkulation ermöglichen. Infrastrukturen pulsieren. Sie sind in rhythmischen Abläufen organisiert.

Transportsysteme bewegen Güter oder Personen in bestimmter Zeit durch den Raum. Damit werden Raum und Zeit rhythmisch strukturiert (Stahl 2018: 144-148). Schon sehr früh haben Schriftsteller wie Walt Whit- 
man beschrieben, wie die gemeinsame Nutzung von öffentlichen Verkehrsmitteln neue soziale Erlebniswelten schuf. Dazu gehörte auch ein Rhythmus gemeinsam verbrachter und erfahrener Zeit etwa von Pendlern (Laak 2018: 245-247). Auch komplexere Systeme wie die aufeinander abgestimmten Ampelschaltungen und Geschwindigkeitsregeln in einer Stadt kreieren Rhythmen, und damit Zeitregime: Auf den Hauptverkehrsadern pulsiert der Verkehr schneller, in den Nebenstraßen bewegen sich die Automobile in niedrigem Tempo. Die Rhythmen der Infrastruktur führen zu Beulen und Dellen im Raum: So rücken gut angeschlossene Punkte (etwa Haltestellen von Hochgeschwindigkeitszügen oder Städte mit Flughäfen) zeitlich nah aneinander, während der nicht angeschlossene ländliche Raum zeitlich in die Ferne rückt (Högselius et al. 2015: 60-63).

Die Rhythmen und Taktungen sind das Ergebnis von planerischen Entscheidungen, aber auch von materiellen Rahmenbedingungen. Beim Aufstellen eines Fahrplanes, etwa der Metro, müssen Bremswege und Beschleunigungszeiten der Bahnen berücksichtigt werden - diese hängen wiederum von Faktoren wie etwa Gewicht, Motorisierung, Signalübertragungswegen, Bremstechnologie ab (Gießmann 2013). Auch die Fließgeschwindigkeiten von Wasser, Gas und Elektronen weichen voneinander ab und generieren damit unterschiedliche Rhythmen in der Versorgungsinfrastruktur. Zur Realität des Infrastrukturbetriebs gehören häufige Unterbrechungen und Betriebsstörungen. Diese verändern die eigentlich vorgesehenen Rhythmen, oft zum Ärger von Nutzern (Zimmer 2019). Damit stellen sie gleichwohl neue Rhythmen, eine so nicht vorgesehene Eigenzeit her (Stahl 2018: 146, 170).

Historisch haben fast alle Infrastrukturen das Ziel, die zeitliche Herrschaft der Natur über den Menschen zu überwinden, also den Menschen von natürlichen Rhythmen unabhängig zu machen. Hier ist die Produktion einer infrastrukturellen Eigenzeit besonders auffällig: Die Beleuchtung von Innenräumen und städtischen Außenräumen mit Gas und Elektrizität seit dem 19. Jahrhundert ermöglichte eine völlig neue Gestaltung von Lebenszeit und sozialer Interaktion (Nye 2018). Der Assuan-Staudamm in Ägypten hat das erklärte Ziel, das Niltal gleichmäßig mit Wasser zu versorgen und die harten Rhythmen der jährlichen Hochwasser zu beenden (Brendel 2019). Anlagen zur Nutzung der Wasserkraft sind darauf ausgelegt, Schwankungen der Pegelstände auszugleichen, um unterbrechungsfreien Betrieb zu gewährleisten (Zumbrägel 2018a: Kap. 5.3).

Aus historischer Perspektive ist vor allem zu betonen, dass die Rhythmen nicht nur Zeit beeinflussen, sondern selbst geschichtlichem Wandel unterliegen. In den zwei Jahrzehnten nach Erreichen der Vollversorgung von Privathaushalten mit Fernsehgeräten, also etwa zwischen 1970 und 1990, gab es ein ausgeprägtes Blockbuster-Phänomen: Bestimmte Fern- 
sehsendungen wurden derart breit rezipiert, dass sich die Straßen leerten. Lebensrhythmus und Nutzung anderer Infrastrukturen waren also stark von der Ausstrahlung bestimmter Sendungen beeinflusst. Mit der Liberalisierung des Mediensektors setzte eine Differenzierung im Freizeitverhalten ein. In der Gegenwart der Streamingdienste trat an die Stelle zentral produzierter Rhythmen individuelle Zeitregime - ein Phänomen, das generell als Merkmal der Netzwerk-Gesellschaften bezeichnet wird (Hassan 2005). Eine ähnliche Entwicklung kann mit Blick auf die New Yorker Untergrundbahn im 20. Jahrhundert beobachtet werden: In den ersten Jahrzehnten ihres Bestehens legten es ihre Betreiber darauf an, die Passagiere mit technischen Anlagen, wie etwa Drehkreuzen, so zu steuern, dass sie sich in bestimmter Geschwindigkeit in bestimmte Richtungen bewegten. Die so geschaffenen Bewegungsrhythmen sollten eine möglichst effiziente Nutzersteuerung und kurze Transportzeiten erzielen. Allerdings kam diese Steuerung bald an ihre Grenzen, Nutzer verweigerten sich oder aber kritisierten die U-Bahn als individualitätsfeindlichen Massenbetrieb (Höhne 2017).

\section{Zeitschichten beeinflussen Rhythmen}

Infrastrukturen sind Ergebnis sedimentierter Vergangenheiten. Sie enthalten unterschiedliche Zeitschichten, die sich in Form technischer Komponenten, Trassenführungen, aber auch in Form von Regularien oder Gesten der Betreiber und Nutzer in die Infrastruktur eingeschrieben haben. Diese Zeitschichten haben wiederum Einfluss auf jene Rhythmen, die eine Infrastruktur in der jeweiligen Gegenwart produziert. Hierzu drei Beispiele: Stellwerke im Eisenbahnbetrieb, dezentrale Energieversorgung um 1900 und Herausforderungen im Leitbild der Smart City.

Ein plastisches Beispiel für die Existenz von Zeitschichten ist die erstaunliche (Alters-)Heterogenität von Stellwerken bei der deutschen Bahn. Es existieren heute nebeneinander Stellwerke mit mechanischem Betrieb (Weichen und Signale werden aus dem Stellwerk per Muskelkraft mit Seilzügen umgestellt), elektromechanischem Betrieb (Signal- und Weichenstellung durch Motoren), Stellwerke mit Relaistechnik (alle Elemente werden auf einem abstrakten Gleisbild dargestellt und durch Tastendruck bedient) und elektronische Stellwerke. Die elektronischen Anlagen werden seit den 1980er Jahren betrieben, die Relaistechnik existiert seit den 1950er Jahren, während einige der mechanischen Anlagen noch aus der Kaiserzeit stammen - in der Summe immerhin noch rund ein Viertel aller Stellwerke. Daher betreibt die Deutsche Bahn auch ein spezielles Ersatzteillager, das 
einem Museum ähnelt. Das sogenannte Signalwerk Wuppertal sammelt gebrauchte Komponenten von Signalen, Weichen und Schaltständen und arbeitet sie für den künftigen Betrieb auf, teilweise mit Technik aus dem frühen 20. Jahrhundert. Das Ersatzteillager ist ein Archiv stellwerktechnologischer Entwicklung seit rund hundert Jahren.

Die Stellwerktechnik ist für Rhythmen verantwortlich: Es sind die Fahrdienstleiter in den Stellwerken, welche die Züge anhalten oder auf ihre Gleisabschnitte schicken - wenn nötig auch in Abweichung vom Fahrplan. Insgesamt arbeitet das System trotz seiner Heterogenität gut integriert, weil die Grundsätze der Zugdisposition sich nicht verändert haben: Weichen und Signale werden so gestellt, dass Züge im sicheren Abstand fahren, also definierte Streckenabschnitte immer nur von einem Zug benutzt werden, um Unfälle zu verhindern. Dennoch arbeiten die Stellwerke aufgrund der technologischen Verschiedenheit sehr unterschiedlich. Viele der mechanischen Anlagen setzen noch heute eine Sichtkontrolle voraus, so dass der Fahrdienstleiter seinen Streckenabschnitt im Wortsinn in Augenschein nehmen muss, bevor eine Freigabe erteilt werden kann. Dies und die mechanische Umstelltechnik mit Seilzügen haben zur Folge, dass mit der alten Technik nur kurze Abschnitte gemanagt werden können. Dagegen funktionieren die jüngeren Stellwerke für deutlich größere Streckenbereiche. Neuerdings können im volldigitalisierten System die Abstände zwischen den Zügen reduziert werden. Die Heterogenität der Technologie führt also dazu, dass Züge in bestimmten Sektoren effizienter und damit schneller abgefertigt werden können, während andere Sektoren gleichsam bremsend wirken. Insgesamt wird der Rhythmus des Bahnverkehrs also von den Zeitschichten der Stellwerkstechnik stark beeinflusst (vgl. Thomas 2013; Strassmann 2019).

Ein weiteres Beispiel ist die Energieerzeugung durch Wasserkraft im Bergischen Land um 1900. Christian Zumbrägel hat in diesem Zusammenhang drei unterschiedliche Zeitregime identifiziert, ohne allerdings diesen Begriff zu verwenden: erstens den Rhythmus täglicher Wartung, zweitens den Rhythmus saisonal-witterungsbedingter Instandhaltung sowie schließlich den episodisch eintretenden Rhythmus umfassender Reparatur infolge von Totalausfällen (Zumbrägel 2018b).

Dieses Beispiel ist deshalb so interessant, weil es einerseits die Abhängigkeit der Rhythmen von Zeitschichten der Infrastruktur zeigt, zum anderen die Verwobenheit von technischen Artefakten mit den sie begleitenden Praktiken (Zumbrägel 2018b: 177-181). Zumbrägel weist darauf hin, dass die drei Rhythmen in dieser klaren Ausprägung das Ergebnis von altersheterogenen technischen Anlagen waren - also in der Terminologie dieses Aufsatzes: das Ergebnis unterschiedlicher Zeitschichten. Zunächst bestanden die Wassermühlen nämlich aus Komponenten höchst unterschiedli- 
chen Alters. Dies lag daran, dass sie immer wieder in Teilen ersetzt, aber kaum komplett ausgetauscht wurden. Die Altersheterogenität der Anlagen war Ursache wie auch Folge häufiger Instandhaltung und Teilreparaturen.

$\mathrm{Zu}$ Beginn des 20. Jahrhunderts jedoch wurden in kurzer Zeit viele neue Anlagen errichtet, die aus einem Guss waren. Sie ersetzten ihre altersheterogenen Vorgänger, womit sich auch die Wartungsrhythmen und damit das Zeitregime der Wasserkraftbetriebe fundamental änderte. Das lag an den verbauten Materialien: Anstelle von Holz kam nun vor allem Metall mit geringerem Verschleiß zum Einsatz. Die tägliche Wartung sowie die saisonale Instandhaltung wurden immer seltener. Bei den Totalausfällen ergaben sich weniger positive Folgen: Brach eine der neuen Mühlen zusammen, so dauerte es lange, bis sie wieder in Betrieb genommen werde konnte. Dagegen war die Reparatur bei den traditionellen altersheterogenen Anlagen vergleichsweise schnell gegangen. Der Grund lag im Material, Holz, welches leichter zu bearbeiten war, aber auch schlicht in der Tatsache, dass die Teile häufiger ausgewechselt wurden.

Hieraus kann man die Hypothese ableiten, dass altersheterogene Systeme mit vielen Zeitschichten tendenziell zu häufigen, dafür aber kurzen Unterbrechungsrhythmen führen. Zeitlich homogenere Systeme begünstigen dagegen länger andauernde Funktionszyklen mit dem Nachteil weniger vorhersehbarer Rhythmisierung von Ausfallzeiten. Das Mühlenbeispiel ist natürlich eingeschränkt und nicht ohne weiteres auf andere Systeme übertragbar. Die Stellwerke der Deutschen Bahn legen aber ähnliche $\mathrm{Zu}$ sammenhänge nahe.

Ein weiteres Beispiel bezieht sich auf eine der jüngeren Entwicklungen in der Infrastrukturgeschichte, nämlich das Leitbild der Smart City (das Beispiel beruht auf der Lektüre von Coletta \& Kitchin 2017; Kitchin 2017a und 2017b). Voraussetzung für Idee und Praxis der Smart City ist der enorme Ausbau der Informationstechnologie, insbesondere des Internet und der Rechnerkapazitäten seit der Jahrtausendwende. Die Smart City ist also aufs Engste verbunden mit einer neuen netzgebundenen Kommunikationsinfrastruktur. Zugleich zielt Smart City darauf ab, Betriebsabläufe in anderen Infrastruktursektoren zu optimieren. Infrastruktur ist somit Voraussetzung und Objekt zugleich von Smart-City-Konzepten. Was hat das mit Zeit zu tun?

Hier sind zwei Gesichtspunkte zu differenzieren. Zum ersten sind Smart-City-Anwendungen vor allem im Verkehrsbereich darauf ausgerichtet, Rhythmen zu verändern und zu optimieren. Rob Kitchin analysierte in seinen Forschungen das aktuelle Verkehrsleitsystem der Stadt Dublin (Coletta \& Kitchin 2017). Mittels tausender Sensoren wird hier das Verkehrsaufkommen kontinuierlich aufgezeichnet und die Ampelschaltungen werden auf dieser Datengrundlage laufend so angepasst, dass 
möglichst wenig Staus entstehen. Die Rhythmen der Ampelphasen sollen also möglichst unterbrechungslose Zirkulation ermöglichen.

Die zweite Beziehung zur Zeit ist eine konzeptuelle. Das Leitbild der Smart City umfasst die Vorstellung, Systeme in Echtzeit zu analysieren und ihr Management dadurch zu optimieren. Nicht historisch ermitteltes Wissen und auch keine Zukunftsprojektionen seien erforderlich für einen erfolgreichen Infrastrukturbetrieb, sondern in erster Linie aktuelle Informationen und sofortige Reaktionen, so zumindest das Versprechen der Protagonisten von Smart City (vgl. Kitchin 2017a: 27). Smart City könnte damit ein Paradebeispiel sein für ein ahistorisches, nur der Gegenwart des Moments verpflichtetes Managementsystem.

Diese Vorstellung ist allerdings eine Illusion. In den Infrastruktursystemen der Smart City sind Zeitschichten ebenso wirksam wie in anderen Systemen. Dafür sprechen mehrere Gründe. Zum ersten gibt es keine Echtzeit in Reinform - jede Information im Leitstand der Dubliner Verkehrsbehörden hat eine Geschichte, und zwar eine doppelte: Zum einen ist sie aufgrund einer historischen Entscheidung ermittelt worden (Platzierung der Sensoren an bestimmten Straßenabschnitten), zum anderen hat jede Messung ein Alter, und seien es nur Millisekunden. Die Steuerung der Ampeln ist also zeitlich immer eine Nachsteuerung. Viel bedeutender sind aber andere historische Umstände: Die Steuerungssoftware mit ihren Algorithmen ist (ähnlich wie die Standortentscheidung für die Sensoren) das Ergebnis von Prioritätensetzungen, die einer bestimmten Epoche mit ihren Werten und Machtverhältnissen entspricht. Für Dublin kann Kitchin feststellen, dass eine stark instrumentelle Rationalität herrscht mit dem Ziel die Rhythmen so zu kalibrieren, dass Unterbrechungen oder Verlangsamungen möglichst vermieden werden. Dies interpretiert er als Ausdruck einer neoliberalen Optimierungs- und Verwertungsideologie, wie sie seit der Jahrtausendwende dominiert (Kitchin 2017a: 31). Wichtig ist die Erkenntnis, dass es sich hier nicht um technologische Notwendigkeiten, sondern um politisch oder kulturell geprägte Auswahlentscheidungen handelt. Weitere Umstände wären zu ergänzen. Selbstverständlich sind für die Ampelschaltungen auch die historischen Trassenentscheidungen konstitutiv: Welche Straßen wo verlaufen, welche Kapazität sie haben, welche Stadtteile also prioritär angeschlossen sind, wie die Verteilung zwischen den Verkehrsträgern angelegt ist und so fort spielt dauerhafte eine große Rolle im Verkehrssystem. So sind also auch die Stop-and-go-Rhythmen in den Straßen der Smart City Dublin ein Ergebnis sedimentierter Zeit. 


\section{Ausblick}

Für Betreiber und Nutzer aktueller Infrastrukturen kann die Perspektive auf Zeit zwei relevante Erkenntnisse bieten. Zum ersten machen die Zeitschichten deutlich, dass die Ursachen für die Komplexität heutiger Infrastrukturen oft weit in der Vergangenheit liegen. Damit können auch Erwartungen und Ansprüche an Veränderungen und Optimierungen im Betrieb realistischer, nämlich bescheidener, eingeschätzt werden. Zugleich erhöht dies die Sensibilität für die Notwendigkeit einer erfolgreichen „Nachsorge" alter Komponenten in bestehenden Infrastruktursystemen (vgl. auch das Plädoyer bei Weber 2019: 142). Umgekehrt kann das historisch informierte Wissen über eine Infrastruktur eine strategische Ressource für ihre Betreiber sein - sogar in Smart Cities.

Zum zweiten sollte bewusster werden, dass die unterschiedlichen Zeitschichten eines Systems Einfluss auf den aktuellen Betrieb haben. Die von einer Infrastruktur produzierten Rhythmen sind auch eine Folge ihrer Geschichtlichkeit. Wenn es stimmt, dass das Herstellen von Zeit immer auch eine Form der Machtausübung darstellt (Coletta \& Kitchin 2017), so liegt sie folglich nicht allein in den Händen der aktuell Handelnden. Die Macht wird dann eben auch vermittelt ausgeübt (vgl. Engels \& Schenk 2014). Historische Zeiten, vermittelt über und materialisiert in den Zeitschichten der Infrastruktur, wirken auf diese Weise bis in die Gegenwart und betreffen jeden Nutzer und jede Ampelschaltung. Allerdings lässt sich diese zeitliche Nachwirkung von Entscheidungen nicht auf Einschränkungen und Pfadabhängigkeiten reduzieren. Dies liegt auch daran, dass häufig viele unterschiedliche Zeitschichten zusammenwirken, viele Systeme im Sinn der Metapher vom Palimpsest also mehrfach überschrieben werden. Infrastrukturen speichern heterogene vergangene Zeiten, und sie beeinflussen die Rhythmen der Gegenwart.

\section{Anmerkungen}

1 Dank an Mark de Bruijne (TU Delft) für den Hinweis.

\section{Literatur}

Ackermann, Felix 2010. Palimpsest Grodno. Nationalisierung und Sowjetisierung einer mitteleuropäischen Stadt 1919-1991. Wiesbaden: Harrassowitz.

Adam, Barbara 1990. Time and Social Theory. Cambridge: Polity Press. 
Ambrosius, Gerold und Christian Henrich-Franke 2013. Integration von Infrastrukturen in Europa im historischen Vergleich. Band 1: Synopse. Baden-Baden: Nomos.

Ambrosius, Gerold und Christian Henrich-Franke 2015. Pfadabhängigkeiten internationaler Infrastrukturnetze. Jahrbuch für Wirtschaftsgeschichte (56): 111-132.

Beyer, Jürgen 2006. Pfadabhängigkeit: Über institutionelle Kontinuität, anfällige Stabilität und fundamentalen Wandel. Frankfurt a.M.: Campus Verlag.

Bijker, Wiebe E. 2007. American and Dutch Coastal Engineering. Differences in Risk Conception and Differences in Technological Culture. Social Studies of Science (37): 143-152.

Bijker, Wiebe E., Thomas P. Hughes und Trevor J. Pinch (Hg.) 1987. The Social Construction of Technological Systems. Cambridge, MA: The MIT Press.

Binder, Julia 2015. Stadt als Palimpsest. Zur Wechselwirkung von Materialität und Gedächtnis. Berlin: Neofelis Verlag.

Blaise, Clark 2000. Time Lord. Sir Sandford Fleming and the Creation of Standard Time. London: Weidenfeld \& Nicolson.

Brendel, Benjamin 2019. Konvergente Konstruktionen. Eine Globalgeschichte des Staudammbaus. Frankfurt a.M.: Campus Verlag.

Champion, Matthew S. 2019. The History of Temporalities. An Introduction. Past and Present (243): 247-254.

Coletta, Claudio and Rob Kitchin 2017. Algorhythmic governance. Regulating the 'heartbeat' of a city using the Internet of Things. Big Data E Society (4/2): 1-16.

David, Paul A. 1986. Understanding the Economics of QWERTY. The Necessity of History. In: William N. Parker (Hg.). Economic History and the Modern Economist. Oxford: Blackwell: $30-49$.

Dolata, Ulrich und Raymund Werle 2007. „Bringing Technology back in“: Technik als Einflussfaktor sozioökonomischen und institutionellen Wandels, in: Ulrich Dolata und Raymund Werle (Hg.). Gesellschaft und die Macht der Technik. Frankfurt a.M.: Campus Verlag: 15-43.

Doyle, Christopher und Raimondo Betti (Hg.) 2010. Aging Infrastructure: Issues, Research, and Technology. Washington: Department of Homeland Security.

Engels, Jens Ivo und Gerrit J. Schenk 2014. Infrastrukturen der Macht - Macht der Infrastrukturen. Überlegungen zu einem Forschungsfeld. In Birte Förster und Martin Bauch (Hg.). Wasserinfrastrukturen und Macht von der Antike bis zur Gegenwart. München: De Gruyter Oldenbourg: 22-58.

Esposito, Fernando (Hg.) 2017. Zeitenwandel. Transformationen geschichtlicher Zeitlichkeit nach dem Boom. Göttingen: Vandenhoeck \& Ruprecht.

Farías, Ignacio und Thomas Bender (Hg.) 2010. Urban Assemblages. How Actor-Network Theory Changes Urban Studies. London: Routledge.

Fryxell, Allegra R.P. 2019. Time and the Modern: Current Trends in the History of Modern Temporalities. Past and Present (243): 285-298.

Geppert, Alexander C. T. und Till Kössler (Hg.) 2015. Obsession der Gegenwart. Zeit im 20. Jahrhundert. Göttingen: Vandenhoeck \& Ruprecht.

Gießmann, Sebastian 2013. Synchronisation im Diagramm. Henry C. Beck und die Londoner Tube Map von 1933. In: Christian Kassung und Thomas Macho (Hg.). Kulturtechniken der Synchronisation. Paderborn: Fink: 339-364.

Graham, Mark 2013. The Virtual Dimension. In: Michele Acuto und Wendy Steele (Hg.). Global City Challenges. Debating a Concept, Improving the Practice. Basingstoke: Palgrave Macmillan: 117-139.

Graham, Stephen und Nigel Thrift 2007. Out of Order. Understanding Repair and Maintenance. Theory, Culture E Society (24/3): 1-25.

Guldi, Jo 2012. Roads to Power. Britain Invents the Infrastructure State. Cambridge: Cambridge University Press.

Hassan, Robert 2005. Timescapes of the Network Society. Fast Capitalism (1): 1-14.

Högselius, Per, Arne Kaijser und Erik van der Vleuten 2015. Europe's Infrastructure Transition. Economy, War, Nature. Basingstoke: Palgrave Macmillan.

Höhne, Stefan 2017. New York City Subway. Die Erfindung des urbanen Passagiers. Köln: Böhlau. 
Hughes, Thomas P. 1983. Networks of Power: Electrification in Western Society 1880-1930. Baltimore: Johns Hopkins University Press.

Kitchin, Rob 2017a. The Realtimeness of Smart Cities. Tecnoscienza (8/2): 19-41.

Kitchin, Rob 2017b. The timescape of smart cities. SocArXiv: 1-35.

Koselleck, Reinhart 2000. Zeitschichten. Studien zur Historik. Frankfurt a.M.: Suhrkamp.

Laak, Dirk van 2001. Infra-Strukturgeschichte. Geschichte und Gesellschaft (27): 367-393.

Laak, Dirk van 2004. Imperiale Infrastruktur. Deutsche Planungen für eine Erschließung Afrikas 1880-1960. Paderborn: Schöningh.

Laak, Dirk van 2018. Alles im Fluss. Die Lebensadern unserer Gesellschaft - Geschichte und Zukunft der Infrastruktur. Frankfurt a.M.: S. Fischer.

Landwehr, Achim 2012. Von der „Gleichzeitigkeit des Ungleichzeitigen“. Historische Zeitschrift (295): 1-34.

Landwehr, Achim 2017. Die vielen, die anwesenden und die abwesenden Zeiten. In: Fernando Esposito (Hg). Zeitenwandel. Transformationen geschichtlicher Zeitlichkeit nach dem Boom. Göttingen: Vandenhoeck \& Ruprecht: 227-253.

Lefebvre, Henri 1992. Éléments de rythmanalyse. Introduction à la connaissance des rythmes. Paris: Éditions Syllepse.

Little, Richard G. 2002. Controlling Cascading Failure: Understanding the Vulnerabilities of Interconnected Infrastructures. Journal of Urban Technology (9): 109-123.

Lübbe, Hermann 2000. Gegenwartsschrumpfung und zivilisatorische Selbsthistorisierung. In: Frithjof Hager und Werner Schenkel (Hg.). Schrumpfungen. Chancen für ein anderes Wachstum. Berlin: Springer: 11-20.

Maassen, Anne 2012. Heterogeneity of Lock-in and the Role of Strategic Technological Interventions in Urban Infrastructural Transformations. European Planning Studies (20): 441-460.

Mahoney, James 2000. Path Dependence in Historical Sociology. Theory and Society (29): 507-548.

Matern, Antje 2016. Beyond Modernity: Urbane Infrastrukturlandschaften in Transformation. In: Antje Matern (Hg.). Urbane Infrastrukturlandschaften in Transformation: Städte - Orte - Räume. Bielefeld: transcript: 9-35.

Mattelart, Armand 2000. Networking the World 1784-2000. Minneapolis: University of Minnesota Press.

May, Jon und Nigel Thrift (Hg.) 2001. Timespace. Geographies of Temporality. London: Routledge.

Mayntz, Renate 1997. Große technische Systeme und ihre gesellschaftstheoretische Bedeutung. In: Renate Mayntz (Hg.). Soziale Dynamik und politische Steuerung. Frankfurt a.M.: Campus Verlag: 70-85.

McFarlane, Colin 2011. Assemblage and critical urbanism. City (15): 204-224.

McTaggart, John McTaggart Ellis 1927. The Nature of Existence. Vol. 2. Cambridge: Cambridge University Press.

Melosi, Martin 2000. The Sanitary City. Urban infrastructure in America from Colonial Times to the Present. Baltimore: Johns Hopkins University Press.

Morin, Gilles 1994. Le canal de l'Est, branche Nord, une infrastructure définitivement obsolète? Revue Géographique de l'Est (1994): 159-166.

Moss, Timothy 2016a. Conserving Water and Preserving Infrastructures between Dictatorship and Democracy in Berlin. Water Alternatives (9): 250-271.

Moss, Timothy 2016b. Discarded surrogates, modified traditions, welcome complements: The chequered careers of alternative technologies in Berlin's infrastructure systems. Social Studies of Science (46): 559-582.

Moss, Timothy 2017. The Rise, Fall and Resurrection of Waste-to-energy Technologies in Berlin's Infrastructure History. In: Niki Frantzeskaki, Vanesa Castán Broto, Lars Coenen und Derk Loorbach (Hg.). Urban Sustainability Transitions. New York: Routledge: $159-171$.

Nowotny, Helga 1992. Time and Social Theory. Towards a Social Theory of Time. Time E Society (1): 421-454.

Nye, David E. 2018. American Illuminations. Urban Lighting 1800-1920. Cambridge, MA: The MIT Press. 
Ogle, Vanessa 2015. The Global Transformation of Time, 1870-1950. Cambridge, MA: Harvard University Press.

Patzel-Mattern, Katja und Albrecht Franz (Hg.) 2015. Der Faktor Zeit. Perspektiven kulturwissenschaftlicher Zeitforschung. Stuttgart: Franz Steiner Verlag.

Perrow, Charles 1984. Normal Accidents. Living with High-Risk Technologies. New York: Basic Books.

Richter, Steffen 2018. Infrastruktur. Ein Schlüsselkonzept der Moderne und die deutsche Literatur 1848-1914. Berlin: Matthes \& Seitz.

Rijkswaterstaat 2017. Vervanging en Renovatie. Prognose voor de periode 2017 tot en met 2050. Den Haag: Rijkswaterstaat.

Roblin, Laurent 2003. Cinq siècles de transport fluvial en France. Du XVIIe au XXIe siècle. Rennes: Éditions Ouest-France.

Rothauge, Caroline 2017. Es ist (an der) Zeit. Zum „temporal turn“ in der Geschichtswissenschaft. Historische Zeitschrift (305): 729-746.

Schenk, Gerrit J. 2012. Managing Natural Hazards. Environment, Society, and Politics in Tuscany and the Upper Rhine Valley in the Renaissance (ca. 1270-1570). In: Andrea Janku, Gerrit J. Schenk und Franz Mauelshagen (Hg.). Historical Disasters in Context. Science, Religion, and Politics. New York/London: Routledge: 31-53.

Schiefelbusch, Martin und Hans-Liudger Dienel (Hg.) 2014. Linking Networks. The Formation of Common Standards and Visions for Infrastructure Development. Farnham: Ashgate.

Schivelbusch, Wolfgang 1977. Geschichte der Eisenbahnreise: Zur Industrialisierung von Raum und Zeit im 19. Jahrhundert. München: Hanser.

Schmolinsky, Sabine, Diana Hitzke und Heiner Stahl (Hg.) 2018. Taktungen und Rhythmen. Raumzeitliche Perspektiven interdisziplinär. Berlin: De Gruyter Oldenbourg.

Schott, Dieter 2018. Krieg als externer Schock oder Katalysator für neue Pfade: Zum Verhältnis von Krieg und Pfadabhängigkeit in deutschen Städten des 20. Jahrhunderts. Moderne Stadtgeschichte (2): 44-58.

Simmie, James 2012. Path dependence and new technological path creation in the Danish wind power industry. European Planning Studies (20): 753-772.

Sprute, Sebastian-Manès 2015. U(h)reigene Zeiten. Grenzen der Implementierung von europäischen Zeitnormen in Senegal, 1890-1930. In: Katja Patzel-Mattern und Albrecht Franz (Hg.). Der Faktor Zeit. Perspektiven kulturwissenschaftlicher Zeitforschung. Stuttgart: Franz Steiner Verlag: 77-105.

Stahl, Heiner 2018. Verkehrsnöte. Rhythmus, Taktung und Störungen des Essener Straßenbahnverkehrs während des Ersten Weltkriegs. In: Sabine Schmolinsky, Diana Hitzke und Heiner Stahl (Hg.). Taktungen und Rhythmen. Raumzeitliche Perspektiven interdisziplinär. Berlin: De Gruyter Oldenbourg: 143-172.

Straßmann, Burkhard 2019. Deutsches Schienennetz. Nachschub aus der Kaiserzeit. Die Zeit Nr. 45/2019, 30. Oktober 2019.

Thomas, Peter 2013. Hundert Jahre Einstellungssache. Frankfurter Allgemeine Zeitung online 24. August 2013.

Thompson, Edward Palmer 1967. Time, Work-Discipline, and Industrial Capitalism. Past and Present (38): 56-97.

Vleuten, Erik van der 2006. Understanding Network Societies: Two Decades of Large Technical System Studies. In: Erik van der Vleuten und Arne Kaijser (Hg.). Networking Europe: Transnational Infrastructure and the Shaping of Europe 1850-2000. Sagamore Beach: Science History Publications: 279-314.

Weber, Heike 2019. Zeitschichten des Technischen: Zum Momentum, Alter(n) und Verschwinden von Technik. In: Martina Heßler und Heike Weber (Hg.). Provokationen der Technikgeschichte. Zum Reflexionsdruck historischer Forschung. Paderborn: Schöningh: 107-144.

Zimmer, Oliver 2019. Die Ungeduld mit der Zeit. Britische und deutsche Bahnpassagiere im Eisenbahnzeitalter. Historische Zeitschrift (308): 46-80.

Zoellner, Tom 2014. Train: Riding the Rails that Created the Modern World. From the TransSiberian to the Southwest Chief. New York: Penguin Books.

Zumbrägel, Christian 2018a. „Viele Wenige machen ein Viel.“ Eine Technik- und Umweltgeschichte der Kleinwasserkraft (1880-1930), Paderborn: Schöningh. 
Zumbrägel, Christian 2018b. Von Mühlenärzten, Turbinenwärtern und Eiswachen: Instandhaltungen am Technikensemble Wasserkraftanlage um 1900. In Stefan Krebs, Gabriele Schabacher und Heike Weber (Hg.). Kulturen des Reparierens. Dinge - Wissen - Praktiken. Bielefeld: transcript: 165-196.

Hinweis des Verlags Der Verlag bleibt in Hinblick auf geografische Zuordnungen und Gebietsbezeichnungen in veröffentlichten Karten und Institutsadressen neutral.

Jens Ivo Engels

Institut für Geschichte

TU Darmstadt

Dolivostraße 15

64293 Darmstadt

Deutschland

engels@pg.tu-darmstadt.de 